\title{
Misdiagnosis of Malaria in Malaria-Dengue Endemic Area
}

\author{
Polrat Wilairatana ${ }^{1,2^{*}}$, Noppadon Tangpukdee ${ }^{1,2}$ and Srivicha Krudsood ${ }^{1,3}$ \\ ${ }^{1}$ Malaria Clinical Research Unit, Malaria Excellence Center, Mahidol University, Thailand \\ ${ }^{2}$ Department of Clinical Tropical Medicine, Mahidol University, Thailand \\ ${ }^{3}$ Department of Tropical Hygiene, Mahidol University, Thailand
}

*Corresponding author: Polrat Wilairatana, Malaria Clinical Research Unit, Malaria Excellence Center and Department of Clinical Tropical Medicine, Faculty of Tropical Medicine, Mahidol University 420/6 Rajvithi Road, Rajthevi, Bangkok 10400, Thailand, Tel: 66-081-8602101; E-mail: polrat.wil@mahidol.ac.th

Received date: June 09, 2014; Accepted date: June 11, 2014; Published date: June 14, 2014

Copyright: (c) 2014 Wilairatana P. This is an open-access article distributed under the terms of the Creative Commons Attribution License, which permits unrestricted use, distribution, and reproduction in any medium, provided the original author and source are credited

\section{Editorial}

In current practice, malaria treatment is carried out after laboratory confirmed diagnosis by microscopy or rapid diagnostic tests (RDTs) [1]. Microscopy is the gold standard and preferred option for diagnosing malaria. In nearly all cases, examination of thick and thin blood films will reveal parasites. Where microscopy is unavailable or unfeasible, RDTs should be used [2]. However in countries where both malaria and dengue are endemic, clinicians may misdiagnose malaria if they do not suspect of malaria in differential diagnosis of febrile illness of the patients and do not request laboratory to confirmative diagnosis of malaria. In Thailand, dengue is endemic in rural and urban areas, whereas malaria is endemic only in rural areas particularly in forests and at international border. Many clinicians did not ask travel history to malaria endemic areas. Many clinicians did not suspect of malaria in febrile patients who routinely live or work in urban areas. Many of them did not think that some febrile urban patients might travel to forests before they had fever. Unlike in the past, the classical malaria fever charts, and teeth-chattering rigors and profuse sweats that characterized the 'paroxysm' are relatively unusual today as symptomatic malaria infections are treated as soon as they are diagnosed. Paroxysms with rigors are more common in vivax and ovale malaria than falciparum and malariae malaria [3]. Some clinicians misunderstand that all malaria patients must have paroxysm. If the febrile malaria patients have no paroxysm, the clinicians may not take the patients' blood for malaria confirmative laboratory diagnosis. Early clinical presentations of malaria and dengue are similar and show non-specific features, eg fever, headache and myalgia. Some clinicians may request only complete blood count (CBC) for investigation of the cause of fever. At presence, many hospitals have used automated machines for CBC. However automated machines cannot detect malaria parasites, if the patients have. Unlike in the past,
$\mathrm{CBC}$ was conducted by manual and the technicians could report malaria parasites if they found in $\mathrm{CBC}$ blood smears despite the clinicians did not request malaria laboratory tests. Thrombocytopenia and atypical lymphocytes could be found in CBCs of both malaria and dengue [4,5]. Clinicians may misdiagnose malaria as dengue due to similar CBC finding by automated machines. In Thailand, many severe malaria patients were misdiagnosed as dengue and had delayed treatment of malaria at early visits to hospitals due to similar nonspecific clinical presentations and similar CBC findings of both diseases. Therefore, in areas where both malaria and dengue are endemic, clinicians should ask travel history of febrile patients and request laboratory confirmative diagnosis of malaria if malaria is suspected.

\section{Acknowledgements}

The authors thank the Office of the Higher Education Commission and Mahidol University under the National Research Universities Initiative, and also the Faculty of Tropical Medicine, Mahidol University, Thailand for kind support.

\section{References}

1. WHO (2010) Guidelines for the treatment of malaria (2nd edn). WHO, Geneva.

2. WHO (2012) Management of severe malaria (3rd edn). WHO, Geneva.

3. Cook GC, Zumla AI (2009) Manson's Tropical diseases, 22nd Edition. Saunders-Elsevier, China.

4. Simon MW (2003) The atypical lymphocyte. Int Pediatr 18: 20-22.

5. Wilairatana P, Tangpukdee N, Muangnoicharoen S, Krudsood S, Kano S (2010) Atypical lymphocytes in malaria mimicking dengue infection in Thailand. Research Reports Trop Med 1: 37-43. 